\title{
JOACHIM UMLAUF
}

\section{Die Germanistik muss sich internationalisieren, um international zu überleben}

Was ist mit der These meines Statements gemeint? Weltweite Verallgemeinerungen sind angesichts der vielen regionalen Unterschiede sicherlich schwierig, doch lässt sich - eben mit Ausnahmen - behaupten, dass das Interesse an Deutsch als Fremdsprache in vielen Ländern rückläufig ist. Einschränkend könnte man hinzufügen: besonders als universitäres Haupt- bzw. Studienfach, besonders in Form traditionell philologischer, literatur- oder sprachwissenschaftlich ausgerichteter Studiengänge. Vielerorts verlagert sich das Interesse auch nur weg von Beschäftigung mit Literatur und Sprache hin zu Fachsprachenerwerb, Deutsch für den Beruf, allgemeiner gefassten Studien über Deutschland, die beispielsweise größere kultur- oder sozialwissenschaftliche Zusammenhänge beleuchten, interdisziplinäre Ansprüche umfassen. Die Frage ist durchaus erlaubt, was „Nationalphilologien", die in einem spezifischen Kontext, dem der Nationenbildung des 19. Jahrhunderts in Europa, entstanden sind, heute angesichts der Globalisierung und eines ,postnationalen“ Europas über die Vermittlung der reinen Sprachkenntnisse hinaus noch zu leisten haben, welche Inhalte sie in ihren Auslandsvarianten noch pflegen sollen. Auslandsvarianten, die bei aller Eigenständigkeit durch eigene Entwicklung, Wissenschaftstraditionen und -diskurse Orientierung bei der Muttergermanistik suchen und in der Vergangenheit auch stets gefunden haben. Bestätigt sich die gegenwärtige Tendenz, die im Ausland vielerorts „Deutschlandstudien“ oder außerhalb unseres Kontinents „Europastudien“" entstehen sieht, die weit über die Kulturwissenschaften hinausreichen, ja manchmal literarische und künstlerische Aus- 
drucksformen nicht mehr berücksichtigen, wirft ein Aspekt dieser Orientierung Probleme auf, weil sich die ausländischen Spezialisten nicht mehr Rat und Impulse bei der Germanistik, sondern zunehmend bei anderen Wissenschaften wie der Geschichtswissenschaft, der Soziologie, der Rechtswissenschaft etc. holen werden. Die Germanistik in Deutschland entwickelt sich, mit vielen kultur- und medienwissenschaftlichen Einsprengseln, die Sprachwissenschaft scheint, was Interdisziplinarität betrifft, vorauszueilen, aber welche Rolle spielt für diese Germanistik die eigene Auseinandersetzung mit der Verfasstheit und der Relevanz des Faches im Ausland? Welche äußere Tragweite und Bedeutung kommt zukünftig der eigenen, sozusagen nach innen gerichteten Arbeit für die ausländischen Germanisten zu? Die Internationalisierung der deutschen Germanistik in diesem Sinne scheint mir unverzichtbar: Hat man sich je systematisch die Frage gestellt, wo die Bedürfnisse der ausländischen Germanistiken liegen? In welchen Bereichen wir Dienstleister unserer Partner werden? Zaghaft beginnen an einigen deutschen Hochschulen die Auseinandersetzungen mit den internationalen Aspekten der Germanistik, zumeist allerdings im Bereich Deutsch als Fremdsprache.

Gewiss, wir werden den Lauf der Welt nicht ändern können, wo insgesamt in Zeiten des Wirtschaftsliberalismus, Forderung nach „Effizienz “ und Drittmitteleinwerbung die ,unproduktiven“ Geisteswissenschaften nicht gerade Hochkonjunktur haben, vielerorts der Begriff der zuweilen berufsunspezifischen Allgemeinbildung zurücksteht hinter berufs- und praxisorientierten Anwendungen. Die deutsche Germanistik sollte sich trotzdem verstärkt für die jeweiligen Situationen in den einzelnen Ländern interessieren und daraus Konsequenzen ziehen, außer sie will sich mit der - zugegebenermaßen essentiellen, aber in der Ausschließlichkeit sterilen - innerdeutschen Rolle als Nationalphilologie bei der Ausbildung von Lehrern etc. zufrieden geben. Aber auch diesbezüglich wäre des öfteren die Frage zu stellen, ob hochspezialisierte Methodendiskurse, in denen manche sich gern ergehen, die nötige, relevante geistige und inhaltliche Orientierung bieten können, mit anderen Worten, ob gerade die Geisteswissenschaften allgemein und die Literaturwissenschaft im Besonderen vielleicht selber Anteil an der vielbeklagten Tatsache haben, dass kulturell-literarischen Erklärungsmodellen zunehmend weniger Aufmerksamkeit und Wertschätzung zukommt. Kann man sich vorstellen, dass aus der Germanistik heraus - sei es direkt oder vermittelt über die Gegenstände ihrer Betrachtung - zu den großen geistigen Themen der Zeit Stellung genommen wird? Und dass solche Stellungnahmen in der Gesellschaft gehört werden? Zu Stammzellen, zum euro-islamischen Dialog, zur europäischen Integration, zur Bedeutung kultureller Toleranz?

Die hier geübte Kritik soll konstruktiv und nicht einseitig verstanden werden, man hätte auch über Auslandsgermanistiken sprechen können, die zu unserem Leidwesen in einigen Ländern - auch oder vorwiegend aus den obengenannten wirtschaftlich-gesellschaftlichen Gründen - in marodem $\mathrm{Zu}$ stand sind, man hätte auch über Geisteswissenschaften oder Philologien ins- 
gesamt debattieren können. Und die hervorragenden Leistungen der Germanistiken im In- und Ausland werden wohl erkannt und anerkannt. Es scheint uns nur, dass bestimmte Dinge vernachlässigt werden, brachliegen, die negative Folgen nach sich ziehen können, mit anderen Worten: die die Krise des Deutschen als Philologie weltweit begünstigen. Und als mit dem akademischen Austausch beauftragte Institution, die umfangreiche Mittel vor allem vom Auswärtigen Amt zur Verfügung gestellt bekommt, die zur Förderung der Germanistik und deutschen Sprache im Ausland dienen, sind wir besonders berufen, uns über geeignete Maßnahmen Gedanken zu machen bzw. den zweckmäßigen Einsatz dieser Mittel einer regelmäßigen Überprüfung zu unterziehen. Dabei kann und will der DAAD keinen Einfluss auf die inhaltliche Entwicklung des Faches ausüben. Stärker als bisher wollen wir jedoch im Lichte der oben vorgebrachten Aspekte die Kontakte und die gemeinsame Reflektion zwischen Inlands- und Auslandsgermanistiken befördern. Im Herbst 2002 veranstalten wir so mit Sondermitteln, die uns das Auswärtige Amt im Rahmen der Beförderung des Dialogs mit islamisch geprägten Ländern zur Verfügung stellt, eine Tagung, zu der wir anerkannte Germanistinnen aus dem Maghreb, dem Nahen Osten und weiteren Ländern dieser Region einladen, um sie mit deutschen Germanisten, aber auch fortgeschrittenen Studierenden ins Gespräch zu bringen, Forschungskontakte zu knüpfen und zu überlegen, was und wie die Germanistik zum Dialog mit diesen Kulturen beitragen kann. Und was die Germanistik in diesen Ländern uns zutragen kann. Diese gemeinsam mit dem Beirat Germanistik im DAAD veranstalteten Begegnungstagungen haben übrigens bereits eine gewisse Tradition. Weitere neue Maßnahmen sind - um nur einige herauszugreifen - im Bereich Fort- und Weiterbildung sowie Kontaktpflege vorgesehen: Dazu können ebenso internationale Sommerakademien zählen, beispielsweise von hochbegabten Nachwuchsgermanisten und Doktoranden aus verschiedenen Ländern, die neben fachlichen Diskussionen Anregungen für die zukünftige Gestaltung ihres Faches und seiner Curricula bekommen sollen, aber auch die verstärkte Betreuung und Schulung von Lektoren, und nicht nur solcher, die vom DAAD vermittelt werden, größere Fachtagungen, die sich beispielsweise mit den theoretischen und wissenschaftlichen Grundlagen von Deutschland- und Europastudien befassen. Auch wenn Deutschlandstudienzentren übrigens in verschiedenen Regionen dieser Welt vermehrt entstehen, diese auch substantiell vom DAAD gefördert werden, heißt das nicht, dass der DAAD der ,traditionellen“ Germanistik den Rücken kehrt. War sie nur früher des öfteren der einzige Ort, wo man sich universitär mit Deutschland und der deutschen Sprache befassen konnte, muss sie sich heute, wo sich die Dinge ausdifferenzieren, zunehmend gefallen lassen, dass nach ihrer Angemessenheit gefragt wird, in Bezug auf ihre Inhalte, aber auch zum Beispiel in Hinsicht auf den Verbleib und die Berufsaussichten ihrer Absolventen. Dabei geht es durchaus nicht um eine Hierarchisierung der studierten Gegenstände oder eine Reduzierung auf ihre pragmatischen Zie- 
le. Politisch ausgerichtete Deutschlandstudien sind nicht mehr wert als die Beschäftigung mit der Literatur des 19. Jahrhunderts, Kulturraumstudien nicht unbedingt sinnvoller als sprachwissenschaftliche Auseinandersetzung. Aber man sollte wissen, warum man die Dinge betreibt. Und uns, wie schon erwähnt, aus deutscher Sicht ständig befragen, was wir beizutragen haben. In verschiedenen Ländern des mittel- und osteuropäischen Geländes ist nationale Identitätsvergewisserung, die bei uns weniger vorrangig erscheint, durchaus aktuell und akut und die Frage nach dem Beitrag der Literatur- oder Sprachwissenschaft dazu ist nicht unsinnig. Die postkoloniale Auseinandersetzung in Indien reflektiert auch in der Literaturwissenschaft in verschiedenen Facetten die Rolle der europäischen Kulturnationen. In Italien ist das Interesse an deutscher Philosophie ungebrochen, in den USA dominieren Partialthemen wie Holocaust, Expressionismus in Film und Kunst oder Gender Studies den geisteswissenschaftlichen Umgang mit Deutschland. In anderen Ländern - als Beispiel sei Frankreich genannt - wo in der Regel nur ein Fach studiert wird, erscheint es immer unattraktiver, ein ganzes Studium einer Sprache und Literatur zu widmen, selbst wenn man mit dem Gedanken spielt, Lehrer zu werden. Zumal die meisten der be- bzw. verhandelten Gegenstände ihre Legitimation aus dem europäischen Bildungskanon ziehen und häufig als Selbstzweck wahrgenommen werden. Ist es wirklich notwendig, den Studierenden des dritten Studienjahres mit Herder zu quälen? Nur, wenn man verständlich macht, warum. So ist in Frankreich der Erfolg der Studiengänge für angewandte Fremdsprachen enorm und dort bröckelt das Deutsche auch noch nicht so ab. Allerdings leiden diese Studiengänge unter dem umgekehrten Exzess: (Zuweilen banalem) Faktenwissen wird absoluter Vorrang eingeräumt, Literatur, Sprachwissenschaft, bildende Kultur und Kunst sind restlos verschwunden. Leider gibt es viele Länder, wo diese Wahl nicht offen steht. Wenn sich die Germanistik darüber klar würde, dass ihre Wahrnehmung über die Grenzen Deutschlands hinausgeht, könnte sie sich auch um die europäische Identitätsfindung verdient machen. Und im übrigen vieles lernen. Das muss sie, wenn sie ihre Relevanz über die Grenzen des deutschsprachigen Raumes hinaus bewahren will. 